\title{
INTERCAMBIABILIDAD DE MEDICAMENTOS MULTIFUENTE EN EL PERÚ: NECESIDAD DE ESTABLECER UNA DIRECTIVA TÉCNICA
}

\author{
Drug interchangeability multi source in peru: the need to establish a technical directive
}

\author{
Katia L. Meza, Lissete D. Monteverde, José R. Juárez \\ Universidad Nacional Mayor de San Marcos
}

\section{RESUMEN}

El presente trabajo, de diseño analítico descriptivo, tiene como objetivo evaluar la necesidad de implementar una Directiva Técnica para estudios que garanticen intercambiabilidad de medicamentos multifuente en el Perú, basados en calidad, eficacia y seguridad. Se propone la intercambiabilidad a través del cumplimiento de Buenas Prácticas de Manufactura, especificaciones de calidad y equivalencia terapéutica, así como, un listado de principios activos que requieren demostrar intercambiabilidad a través de estudios in vivo in vitro. Se concluye que existe la necesidad de una reglamentación que asegure la intercambiabilidad de los medicamentos multifuente, que permita actualizar los sistemas de regulación conforme a los programas de armonización internacional, reduzca el gasto en salud pública proporcionando medicamentos de acceso universal, garantice la calidad, eficacia y seguridad de los mismos y genere mayor confiabilidad respecto a la calidad de nuestros productos.

Palabras clave: Intercambiabilidad, bioequivalencia, equivalencia terapéutica, medicamentos genéricos, productos multifuente, bioexenciones.

\section{SUMMARY}

This descriptive and analytical work, aims to evaluate the need to implement a Technical Directive for studies that ensure interchangeability of multisource drugs in Peru, based on quality, efficacy and safety. It is suggested the interchangeability through compliance with Good Manufacturing Practices, quality specifications and therapeutic equivalence, as well as, a list of active ingredients that had proven to be interchangeable in vivo and in vitro studies. We conclude that regulation is needed to ensure the interchangeability of multisource drug, that permit update regulation systems according to international harmonization programs, ensure quality, efficacy and safety of this drug, reduce public health spending providing medicines universal access and generate greater confidence regarding the quality of our products.

Keywords: Interchangeability, bioequivalence, therapeutic equivalence, generic medicine, multisource products, biowaivers.

\section{INTRODUCCIÓN}

a oferta del mercado farmacéutico, es tanto por medicamentos innovadores, genéricos y similares o multifuente. La industria de medicamentos genéricos continúa teniendo la mayor participación en los mercados del mundo, haciéndolos más accesibles a la población ${ }^{(1)}$. El análisis del mercado farmacéutico en el Perú, evidencia el alto precio promedio de los medicamentos y el bajo interés de los profesionales de la salud por la calidad del medicamento multifuente, originando un consumo aproximado de $11 \%$ en el mercado privado, mientras que en otro países alcanza el $50 \%$ o más ${ }^{(2)}$.

El concepto de medicamento genérico conlleva la idea de "intercambiabilidad" con un medicamento de referencia ${ }^{(1)}$, asegura una oferta de calidad, eficaz y segura, promoviendo mayor confianza en su uso.

La intercambiabilidad puede ser definida en tres tipos: obvia, atribuible a los requerimientos de Buenas
Prácticas de Manufactura y especificaciones técnicas de calidad; demostrada por ensayos in vitro o in vivo ${ }^{(3)}$.

En el Perú no existe legislación que exija estudios para garantizar la intercambiabilidad, la cual se establece como criterio para la obtención del registro sanitario, según el reglamento de productos farmacéuticos ${ }^{(4,5)}$, por lo que el cumplimiento de éste requisito es aún incierto. Por eso planteamos como objetivo la necesidad de implementar una Directiva Técnica que establezca los criterios para la exigencia de estudios que garanticen la intercambiabilidad de los medicamentos multifuente en el Perú, asegurando su calidad, eficacia y seguridad.

\section{MATERIAL Y MÉTODOS}

\section{Materiales}

Se utilizaron, como referencia, documentos sobre políticas de medicamentos genéricos, guías internacionales de bioequivalencia, criterios de 
bioexención de la Organización Mundial de Salud $(\mathrm{OMS})^{(6)}$ y de agencias regulatorias de medicamentos, como Food and Drug Administration (FDA), Agencia Europea de Medicamentos (EMA), Grupo de Trabajo de Bioequivalencia (GT/BE) Armonización Farmacéutica para las Américas 2001-2009 (OMS, OPS, FDA, y países de la Región LA), Agencia Nacional de Vigilancia Sanitaria (ANVISA) de Brasil, Administración Nacional de Medicamentos Alimentos y Tecnología (ANMAT) de Argentina, Instituto Nacional de Vigilancia de Medicamentos y Alimentos (INVIMA) de Colombia, Instituto de Salud Pública (ISP) de Chile, Organización Panamericana de Salud (OPS) ${ }^{(3,7)}$, Dirección General de Medicamentos Insumos y Drogas (DIGEMID) del Perú ${ }^{(5)}$.

Información complementaria, sobre datos estadísticos en salud y medicamentos, fue proporcionada por consulta directa y vía on-line con el Instituto Nacional de Estadística e Informática (INEI) y el IMS Institute for Healthcare Informatics.

\section{Método y procesamiento de la información}

Se realizó una búsqueda exhaustiva y estructurada de las fuentes información antes mencionadas, por internet o solicitud directa, a las autoridades de las agencias reguladoras de medicamentos y empresas farmacéuticas.

\section{RESULTADOS}

1. Análisis de la normativa nacional. En la tabla 1, se muestran las diferentes disposiciones normativas disponibles en el Perú, no encontrándose una reglamentación para la intercambiabilidad como requerimiento para la obtención del Registro Sanitario.

2. Análisis de precios de Medicamentos genéricos en el Perú. En la tabla 2 se presentan algunos precios referenciales de medicamentos multifuente y similares de marca o innovador para ilustrar la diferencia de precios que existe entre ambos grupos.

3. Comparación y análisis de la normativa internacional. Se hizo la revisión de las reglamentaciones de 5 países: Argentina, Chile, Brasil y Estados Unidos; observándose que desde 1999, Argentina y Brasil cuentan con legislación sobre estudios de bioequivalencia, y Chile desde el 2005.

\section{Propuesta de directiva técnica}

Basado principalmente en las guías propuestas por la OMS, FDA, EMA, OPS y las normativas vigentes de países como Chile, Brasil y Argentina, se ha propuesto una Directiva Técnica que señala las disposiciones generales y específicasaconsiderarparaasegurarlaintercambiabilidad. Así, dependiendo de la forma farmacéutica y del sistema de clasificación biofarmacéutica, se opta por estudios in

Tabla 1. Normativas disponibles en Perú referente a medicamentos y aseguramiento de su calidad.

\begin{tabular}{|c|c|c|}
\hline Legislación vigente & Descripción & Publicación \\
\hline Decreto Ley N² 25596 & $\begin{array}{l}\text { Establecen los requisitos para la obtención del Registro Sanitario } \\
\text { y de la autorización para la importación y comercialización de } \\
\text { medicamentos genéricos y de marca. }\end{array}$ & Julio, 1992 \\
\hline Ley $N^{\circ} 26842$ & Ley General de Salud. & Julio, 1997 \\
\hline Decreto Supremo Nº10-97-SA & $\begin{array}{l}\text { Reglamento para el registro, control y vigilancia sanitaria de } \\
\text { productos farmacéuticos y sus modificatorias (Decretos Supremos } \\
N^{\circ} 004-2000-S A, N^{\circ} 006-2001-S A, N^{\circ} 020-2001-S A \text { y N005-2004- } \\
\text { SA, 021-2006). }\end{array}$ & Diciembre, 1997 \\
\hline Resolución Ministerial N055-99-SA/DM - & $\begin{array}{l}\text { Manual de Buenas Prácticas de Manufactura de Productos } \\
\text { Farmacéuticos. }\end{array}$ & Febrero, 1999 \\
\hline Resolución Ministerial N 433-2001-SA/DM & $\begin{array}{l}\text { Dicta normas relativas al control y vigilancia de productos } \\
\text { farmacéuticos y afines. }\end{array}$ & Julio, 2001 \\
\hline Resolución Ministerial N 134-2006-MINSA & $\begin{array}{l}\text { Aprueban Reglamento para la Autorización Sanitaria de Productos } \\
\text { Farmacéuticos y Afines. }\end{array}$ & Febrero, 2006 \\
\hline Decreto Supremo N 017-2006-SA & $\begin{array}{l}\text { Aprueban el Reglamento de Ensayos Clínicos y sus modificaciones } \\
\text { (D.S N006-2007) }\end{array}$ & Julio del 2006 \\
\hline Ley $N^{\circ} 29459$ & $\begin{array}{l}\text { Ley de los Productos Farmacéuticos, Dispositivos Médicos y } \\
\text { Productos Sanitarios. }\end{array}$ & Noviembre, 2009 \\
\hline Decreto Supremo N 028-2010-SA & $\begin{array}{l}\text { Regulan algunos alcances de los Artículos } 10 \text { y } 11 \text { de la Ley N } 29459 \\
\text { Ley de Productos Farmacéuticos, Dispositivos Médicos y Productos } \\
\text { Sanitarios. }\end{array}$ & Setiembre, 2010 \\
\hline Decreto Supremo Nº16-2011/SA & $\begin{array}{l}\text { Aprueban Reglamento para el Registro, Control y Vigilancia Sanitaria } \\
\text { de Productos Farmacéuticos, Dispositivos Médicos y Productos } \\
\text { Sanitarios y su modificatoria Decreto Supremo N } 001-2012 / S A \text {. }\end{array}$ & $\begin{array}{l}\text { Julio, } 2011 \\
\text { Enero, } 2012\end{array}$ \\
\hline Resolución ministerial N 599-2012 & Aprueba el Petitorio Nacional Único de Medicamentos Esenciales. & Julio, 2012 \\
\hline Decreto Supremo Nº14-2011/SA & $\begin{array}{l}\text { Aprueban Reglamento de Establecimientos Farmacéuticos y su } \\
\text { modificatoria Decreto Supremo } \mathrm{N}^{\circ} 002-2012 / \mathrm{SA} \text {. }\end{array}$ & $\begin{array}{l}\text { Julio, } 2011 \\
\text { Enero, } 2012\end{array}$ \\
\hline
\end{tabular}


Tabla 2. Comparación de precios de algunos medicamentos.

\begin{tabular}{lcccc}
\hline \multirow{2}{*}{ Denominación común internacional } & $\begin{array}{c}\text { Fármacos } \\
\text { disponibles }\end{array}$ & $\begin{array}{c}\text { Precio promedio del medicamento } \\
\text { Innovador / } \\
\text { Similares de marca }\end{array}$ & $\begin{array}{c}\text { Multifuente } \\
\text { DCI }\end{array}$ & $\begin{array}{c}\text { Variación } \\
\text { (\%) }\end{array}$ \\
\hline Amoxicilina (tabletas/cápsula 500 mg) & 18 & 2,217 & 0,966 & 130 \\
Captopril (tableta 25 mg) & 10 & 1,575 & 0,625 & 152 \\
Ciprofloxacina (tabletas 500 mg) & 51 & 7,163 & 2,190 & 227 \\
Diazepan (tableta 10 mg) & 6 & 1,300 & 0,259 & 402 \\
Dicloxacilina (tableta 500 mg) & 13 & 2,743 & 1,323 & 107 \\
Fluconazol (cápsula 150 mg) & 30 & 39,280 & 16,054 & 145 \\
Ibuprofeno (tableta 400 mg) & 22 & 1,694 & 0,392 & 332 \\
Naproxeno (tableta 550 mg) & 37 & 2,538 & 1,186 & 114 \\
Ranitidina (tableta 300 mg) & 12 & 3,647 & 1,477 & 147 \\
Sulfametozaxol + trimetropina (tableta 800/160mg) & 28 & 3,322 & 0,690 & 381 \\
\hline
\end{tabular}

Fuente: Elaboración propia. Datos obtenidos sobre la información proporcionada por DIGEMID y datos disponibles en la revista Kairos (2012)

Tabla 3. Medicamentos a los que se debe exigir estudios de bioequivalencia, según riesgo sanitario.

\begin{tabular}{|c|c|c|c|c|c|c|c|c|c|c|c|c|c|}
\hline Producto & Clase terapéutica & SCB & $\begin{array}{l}\text { Riesgo } \\
\text { sanitario }\end{array}$ & 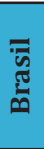 & 를 & $\begin{array}{l}\frac{\pi}{0} \\
\\
\frac{\pi}{2} \\
0 \\
0\end{array}$ & 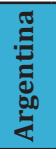 & 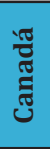 & 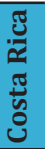 & $\stackrel{\pi}{3}$ & 疍 & 递 & 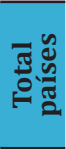 \\
\hline Carbamazepina & $\begin{array}{l}\text { Anticonvulsionantes, } \\
\text { Antiepilépticos, }\end{array}$ & II & 3 & $\mathrm{x}$ & $\mathrm{x}$ & $\mathrm{x}$ & $\mathrm{x}$ & $\mathrm{x}$ & $\mathrm{x}$ & $\mathrm{x}$ & $\mathrm{x}$ & $\mathrm{x}$ & 9 \\
\hline Ciclosporina & Inmunosupresores & II & 3 & $\mathrm{x}$ & $\mathrm{x}$ & $\mathrm{x}$ & $\mathrm{x}$ & $\mathrm{x}$ & $\mathrm{x}$ & $\mathrm{x}$ & $\mathrm{x}$ & $\mathrm{x}$ & 9 \\
\hline Digoxina & Antiarrítmicos & I & 3 & $\mathrm{x}$ & $\mathrm{x}$ & $\mathrm{x}$ & $\mathrm{x}$ & $\mathrm{x}$ & $\mathrm{x}$ & $\mathrm{x}$ & $\mathrm{x}$ & $\mathrm{x}$ & 9 \\
\hline Etambutol clorhidrato & Antituberculosos & III & 3 & $\mathrm{x}$ & & $\mathrm{x}$ & & $\mathrm{x}$ & & $\mathrm{x}$ & $\mathrm{x}$ & $\mathrm{x}$ & 5 \\
\hline Etosuximida & $\begin{array}{l}\text { Anticonvulsionantes, } \\
\text { Antiepilépticos }\end{array}$ & III & 3 & $\mathrm{x}$ & & $\mathrm{x}$ & $\mathrm{x}$ & $\mathrm{x}$ & & & $\mathrm{x}$ & & 4 \\
\hline Fenitoína sódica & $\begin{array}{c}\text { Anticonvulsionantes, } \\
\text { Antiepilépticos }\end{array}$ & II & 3 & $\mathrm{x}$ & $\mathrm{x}$ & $\mathrm{x}$ & $\mathrm{x}$ & $\mathrm{x}$ & $\mathrm{x}$ & $\mathrm{x}$ & $\mathrm{x}$ & $\mathrm{x}$ & 9 \\
\hline Griseofulvina & Antifúngicos & II, IV & 3 & $\mathrm{x}$ & & & & $\mathrm{x}$ & & & $\mathrm{x}$ & & 3 \\
\hline Litio carbonato & trastornos bipolares & I & 3 & $\mathrm{x}$ & $\mathrm{x}$ & $\mathrm{x}$ & $\mathrm{x}$ & $\mathrm{x}$ & & $\mathrm{x}$ & $\mathrm{x}$ & $\mathrm{x}$ & 8 \\
\hline Teofilina & Antiasmáticos & IV & 3 & $\mathrm{x}$ & $\mathrm{x}$ & $\mathrm{x}$ & $\mathrm{x}$ & $\mathrm{x}$ & & $\mathrm{x}$ & $\mathrm{x}$ & $\mathrm{x}$ & 8 \\
\hline Valproato sódico & $\begin{array}{l}\text { Anticonvulsionantes, } \\
\text { Antiepilépticos }\end{array}$ & I & 3 & $\mathrm{x}$ & $\mathrm{x}$ & $\mathrm{x}$ & $\mathrm{x}$ & $\mathrm{x}$ & $\mathrm{x}$ & $\mathrm{x}$ & $\mathrm{x}$ & $\mathrm{x}$ & 9 \\
\hline Verapamilo clorhidrato & Antiarrítmicos & II & 3 & $\mathrm{x}$ & $\mathrm{x}$ & $\mathrm{x}$ & $\mathrm{x}$ & $\mathrm{x}$ & $\mathrm{x}$ & $\mathrm{x}$ & $\mathrm{x}$ & $\mathrm{x}$ & 9 \\
\hline Warfarina sódica & $\begin{array}{l}\text { Que afectan la } \\
\text { coagulación }\end{array}$ & I & 3 & $\mathrm{x}$ & & $\mathrm{x}$ & $\mathrm{x}$ & $\mathrm{x}$ & & $\mathrm{x}$ & $\mathrm{x}$ & $\mathrm{x}$ & 7 \\
\hline
\end{tabular}

Fuente: Elaboración propia.

Tabla 4. Medicamentos a los que se debe evaluar estudios de bioequivalencia según SCB.

\begin{tabular}{cccc}
\hline Producto & Producto & Producto & Producto \\
\hline Acetazolamida & Diloxanida furoato & Etopósido & Nistatina \\
Ácido fólico & Efavirenz & Glibenclamida & Oxicodona clorhidrato \\
Albendazol & Eritromicina & Haloperidol & Pirimetamina \\
Azatioprina & Espironolactona & Indinavir & Retinol palmitato \\
Azitromicina & Sulfasalazina & Ivermectina & Ritonavir \\
Cefuroxima & Triclabendazol & Mefloquina & Saquinavir \\
Clofazimina & Furosemida & Nelfinavir & \\
\hline
\end{tabular}

Fuente: Elaboración propia.

vivo o in vitro. Esta Directiva también incluye un listado de principios activos considerados como prioritarios para exigir el cumplimiento de pruebas específicas que garanticen la equivalencia terapéutica.

\section{DISCUSIÓN}

Las grandes agencias regulatorias recomiendan la demostración de equivalencia terapéutica y la declaración deintercambiabilidad detodos los productos multifuente.
La revisión de la normativa internacional permite visualizar que la realidad de algunos países en vías de desarrollo, es similar a la peruana; sin embargo, presentan avances en la implantación de una política de medicamentos genéricos y exigencias para la realización de estudios de equivalencia terapéutica. Se puede apreciar que muchos países tienen implementada una política de control de precios que favorece y mejora la accesibilidad a los medicamentos esenciales por parte de la población.

En el Perú, los requerimientos para asegurar la intercambiabilidad de productos multifuente no se encuentran bien definidos, por lo que existe la necesidad de establecer lineamientos claramente definidos que garanticen la calidad y accesibilidad a estos medicamentos, además de una política de control de precios y que asegure su intercambiabilidad.

La tabla 2, presenta información de precios de medicamentos en el Perú que considera a los multifuente DCI en comparación a medicamentos innovadores y similares, reflejándose desbalance en el control de precios. Un estudio realizado por la AIS ${ }^{(8)}$, determinó que los países en vías de desarrollo pagan precios más altos que en los países industrializados por los mismos medicamentos. De igual manera, un análisis de los de medicamentos en el Perú ${ }^{(1)}$, comparado con Colombia y Chile, demostró que el consumo promedio anual es menor, pero los precios promedio de los 
medicamentos de marca y genéricos consumidos son mayores. Es evidente que en países similares se están pagando precios distintos por el mismo medicamento, lo que constituye una herramienta poderosa para que las autoridades puedan iniciar una evaluación de precios, de manera que, pueda determinarse la principal razón de dichas diferencias.

Otro punto a debatir, es la gran participación en el mercadofarmacéuticodemedicamentossimilaresdemarca, que a pesar de no tener un perfil comprobado de eficacia y seguridad, al igual que los multifuente DCI, tienen un precio bastante elevado. Con la finalidad de determinar por qué la población prefiere medicamentos similares de marca e innovadores, se buscó información sobre la ineficacia de los medicamentos multifuente con DCI, no encontrándose un registro organizado, lo que haría necesario que DIGEMID plantee un programa de reportes de ineficacias que ayude a identificar aquellos de baja calidad.

Debido a la gran variedad de principios activos comercializados en el país, se ha considerado un programa de implementación basado en 4 criterios: Nivel de riesgo sanitario, Exigencia sanitariadeestudiosdebioequivalencia en otros países, Sistema de clasificación biofarmacéutica y Registro epidemiológico; con los cuales se clasificaron los medicamentos incluidos en el Petitorio Nacional Único de Medicamentos Esenciales. Así, en función al riesgo sanitario, se ha determinado que son 12 los fármacos con estrecho margen terapéutico que requieren estudios de bioequivalencia (tabla 3). De entre estos, se determinará la prioridad con que serán auditados, según: exigencia en otros países y tasa de morbilidad en el Perú.

Teniendo en cuenta estos planteamientos, se propone exigir estudios que demuestren la intercambiabilidad in vivo a: carbamazepina, ciclosporina, digoxina, fenitoína sódica, teofilina, valproato sódico y verapamilo clorhidrato. También, es preciso considerar la necesidad de estudios de equivalencia in vivo a los medicamentos de la tabla 4 , por pertenecer a la clase 4 según SCB. Adicionalmente, se podrían aplicar estudios in vitro a medicamentos como: amoxicilina, ciprofloxacino, cloranfenicol, doxiciclina, isoniazida, mercaptopurina, metotrexato, nitrofurantoína, procarbazina, rifampicina y tamoxifeno.

\section{CONCLUSIÓN}

El Perú no cuenta con una legislación que garantice la intercambiabilidad de productos multifuente, por lo que es necesario implementar una directiva para garantizar la intercambiabilidad a fin de actualizar los sistemas de regulación —conforme a los programas de armonización internacional-, garantizar la calidad, eficacia y seguridad de los productos multifuente que se comercializan, reducir el gasto de salud pública proporcionando medicamentos de acceso universal con múltiples alternativas de elección y generar, finalmente, mayor confiabilidad respecto a la calidad de nuestros productos.

\section{REFERENCIAS BIBLIOGRÁFICAS}

1. Aravena V, Calero C, Martínez O, Navarro M, Villareal R. Desarrollo del medicamento genérico en el Perú. Editorial Cordillera - Universidad ESAN. Lima, 2008.

2. IMS. Venta de medicamentos genéricos en el mundo y crecimiento de ventas en el Perú. [Consulta en línea por correo: Patricia Ramírez; PRamirez@pe.imshealth.com]. Consulta realizada el 15 de febrero del 2012.

3. Villar A, Valladares G, Castro J, Flores V, Quispe P, Arisaca C. Asistencia técnica para la Elaboración de la Propuesta de estrategias para medicamentos Genéricos. Lima, Organización Panamericana de la Salud; 2010.

4. Placencia MD. La Bioequivalencia como requisito de calidad de los medicamentos genéricos/multifuente: estudio comparativo en países latinoamericanos. [Tesis doctoral]. Facultad de Farmacia y Bioquímica. Universidad Nacional Mayor de San Marcos. 2010.

5. Reglamento para el Registro, Control y Vigilancia Sanitaria de Productos Farmacéuticos, Dispositivos médicos y Productos Sanitarios. Decreto Supremo $\mathrm{N}^{\circ}$ o16-2011-SA. Diario El Peruano. Publicado el 27 de Julio del 2011. Lima. [En línea] último acceso 20 de enero de 2012. Disponible en: http://www.digemid.minsa.gob.pe/ normatividad/DSo16-2011-MINSA.pdf

6. World Health Organization. WHO Expert Committee on Specifications for Pharmaceutical Preparations. Fortieth Report. WHO Technical Report Series 937. Geneva, 2006.

7. Organización Panamericana de la Salud. Guía para la implementación de Estrategia de Medicamentos genéricos en los países de América Latina y el Caribe como mecanismo para mejorar el acceso a medicamentos. Documento $\mathrm{N}^{\circ} 3$ Serie Técnica de medicamentos esenciales, Políticas farmacéuticas. OPS. Washington DC, 2011.

8. Acción Internacional para la Salud. Precios de medicamentos en América Latina. Oficina de coordinación AIS LAC. Lima, 2001.

\section{Correspondencia}

Nombre: José R. Juárez

Dirección: Jr. Puno 1002 - Lima 1

E-mail:_ jjuarez@unmsm.edu.pe 